University of Nebraska - Lincoln

DigitalCommons@University of Nebraska - Lincoln

$5-13-2002$

\title{
Threshold-Related Enhancement of the High-Energy Plateau in Above-Threshold Detachment
}

\author{
Bogdan Borca \\ University of Nebraska - Lincoln \\ M.V. Frolov \\ Voronezh State University, Voronezh, Russia \\ N.L. Manakov \\ Voronezh State University, manakov@phys.vsu.ru \\ Anthony F. Starace \\ University of Nebraska-Lincoln, astarace1@unl.edu
}

Follow this and additional works at: https://digitalcommons.unl.edu/physicsstarace

Part of the Physics Commons

Borca, Bogdan; Frolov, M.V.; Manakov, N.L.; and Starace, Anthony F., "Threshold-Related Enhancement of the High-Energy Plateau in Above-Threshold Detachment" (2002). Anthony F. Starace Publications. 84.

https://digitalcommons.unl.edu/physicsstarace/84

This Article is brought to you for free and open access by the Research Papers in Physics and Astronomy at DigitalCommons@University of Nebraska - Lincoln. It has been accepted for inclusion in Anthony F. Starace Publications by an authorized administrator of DigitalCommons@University of Nebraska - Lincoln. 


\title{
Threshold-Related Enhancement of the High-Energy Plateau in Above-Threshold Detachment
}

\author{
Bogdan Borca, ${ }^{1, *}$ M. V. Frolov, ${ }^{2}$ N. L. Manakov, ${ }^{2}$ and Anthony F. Starace ${ }^{1}$ \\ ${ }^{1}$ Department of Physics and Astronomy, The University of Nebraska, Lincoln, Nebraska 68588-0111 \\ ${ }^{2}$ Research and Education Center, Voronezh State University, 394693 Voronezh, Russia
}

(Received 18 October 2001; published 24 April 2002)

\begin{abstract}
We present nonperturbative theoretical results showing a resonant-like enhancement of abovethreshold detachment spectra in the region of the high-energy plateau as the laser intensity sweeps across channel thresholds. This enhancement has a pure quantum origin stemming from well-known threshold phenomena in multichannel problems whose features are clearly demonstrated in our numerical results. Similar well-known anomalies at neutral atom thresholds are expected to explain experimentally observed resonant-like enhancements of above-threshold ionization spectra.
\end{abstract}

DOI: 10.1103/PhysRevLett.88.193001

A fruitful process for understanding the interaction of intense laser light with atoms has been above-threshold ionization (ATI), in which the ionized electron spectrum exhibits a series of peaks, separated in energy by the photon frequency, and having nearly equal intensity over large energy regions (the so-called "plateau" region) $[1,2]$. ATI phenomena present a challenge to theory owing to the necessity of a nonperturbative treatment. Nevertheless many key features of ATI spectra have been understood experimentally or by using classical, semiclassical, or approximate quantum mechanical analyses and calculations [1,2]. These include the role of Rydberg resonances in producing the structure of individual (generally lower energy) ATI peaks and the role of rescattering of the electron by the ionic core in determining the extent of the high-energy plateau. Recently, however, a resonant-like enhancement of ATI peak intensities in the high-energy plateau has been discovered experimentally $[3,4]$. It does not seem to be related to the Rydberg structure of neutral atoms and is very sensitive to the laser intensity $[3,4]$. Nor is it related to two-electron resonances; indeed, a time-dependent, single active electron calculation is able to reproduce it, showing that a 5\% change of laser intensity can result in an order of magnitude change in the relevant ATI peak intensities [5]. Reference [5] attributes the ATI peak enhancements to resonant-like constructive interference between rescattered ionized electron wave packets produced in different laser field cycles. Calculations employing a zero-range potential (ZRP) have demonstrated similar enhancement in the related above-threshold detachment (ATD) spectrum, including the great sensitivity to laser intensity and the necessity of including rescattering effects [6]. Reference [6] attributes the enhancement to constructive interference of electron trajectories as the laser intensity varies [6]. Most recently, the role of channel closings (CCs), i.e., threshold effects, have been identified as important for describing the observed enhancement in experiments that used very short laser pulses of $50 \mathrm{fs}$ [7] and $40 \mathrm{fs}$ [8]. The experiments in [7] were simulated by ZRP calculations; the resonant-like behavior of peaks along the high-energy plateau as a function of laser intensity was interpreted as being due to con-
PACS numbers: $32.80 . \mathrm{Rm}, 32.80 . \mathrm{Gc}, 32.80 . \mathrm{Qk}$

structive interference of many active electron trajectories near multiphoton channel closings [7]. More realistic calculations for Ar were carried out for the experiments in [8], and the resonant-like behavior was interpreted as being due to $n$-photon resonant enhancement of higher channels near the $n$-photon channel closing [8]. Despite these works, the precise nature of this ATI resonant-like effect remains, as characterized in 1998 by Muller and Kooiman [5], a "mystery."

In this Letter we present essentially exact numerical results for high-order multiphoton detachment from a ZRP that identify the resonant-like enhancement of high-energy ATD peaks as stemming from general analytic features of partial cross sections at the thresholds of particular channels. Specifically, at the closing of even photon channels involving $l=0$ electrons, analytically known cusp features appear in higher photon ATD channels [9]. The sensitive dependence of the channel thresholds to the laser intensity results in a corresponding sensitivity of ATD peak intensities. While the effect of threshold anomalies (TAs) at $\mathrm{CCs}$ has been noted for other strong field processes (e.g., closing of stimulated electron bremsstrahlung channels on ATD spectra [10], and closing of ATD channels on high-order harmonic generation spectra [11]), these prior studies do not address the broad, resonant-like enhancement of the high-energy ATD plateau. Finally, although we focus on ATD, we discuss also the applicability of our results to understanding past experimental works for neutral atoms $[3,4,7,8]$, which we regard as remarkable experimental demonstrations of the well-known TAs of general scattering theory $[9,12,13]$ for the case of multiphoton processes.

Our analysis is based on ab initio results obtained using a nonperturbative quasistationary quasienergy state (QQES) theory [14] for detachment of an electron bound in a three-dimensional ZRP. This theory has been discussed in detail elsewhere $[15,16]$ and has been used to describe other intense field processes [17,18]. For the ZRP model, the key quantities of the QQES approach are the quasienergy $\epsilon=E_{0}+\Delta-i \Gamma / 2$, where $\Delta$ is the Stark shift of the initial level $E_{0}$ and $\Gamma$ is its decay rate, and 
the Fourier coefficients $\phi_{s}$ of the periodic QQES wave function $\Phi_{\epsilon}(\mathbf{r}, t)$ for $r \rightarrow 0$ [15]. We assume the electron interacts with a linearly polarized laser field, $\mathbf{F}(t)=$ $F \hat{\boldsymbol{\epsilon}} \cos \omega t$, where $F$ is the laser amplitude, $\hat{\boldsymbol{\epsilon}}$ is the polarization unit vector, and $\omega$ is the frequency. The $n$-photon detachment amplitude, $\mathcal{A}_{n}$, for electron ejection in the direction $\mathbf{n}$, and the $n$-photon rate, $\Gamma^{(n)}$, are given by [18]

$$
\begin{gathered}
\Gamma^{(n)}=2 \int\left|\sqrt{k_{n}} \mathcal{A}_{n}\right|^{2} d \Omega_{\mathbf{n}}, \\
\mathcal{A}_{n}=\sum_{s=-\infty}^{\infty}(-1)^{s} \phi_{s} J_{2 s+n}\left(\frac{2 F k_{n}}{\omega^{2}}(\hat{\boldsymbol{\epsilon}} \cdot \mathbf{n})\right) .
\end{gathered}
$$

Here $k_{n}$ is the complex "momentum," $k_{n} \equiv$ $\sqrt{\epsilon+n \omega-U_{p}}$, and $U_{p} \equiv F^{2} /\left(2 \omega^{2}\right)$ is the ponderomotive shift. (In order to apply our results to any atomic system, we employ scaled units, in which energies and $\hbar \omega$ are in units of $\left|E_{0}\right|$ and $F$ is in units of $F_{0}=$ $\sqrt{2 m\left|E_{0}\right|^{3}} /|e| \hbar$; e.g., for $\mathrm{H}^{-},\left|E_{0}\right|=0.754 \mathrm{eV}$ and $F_{0}=$ $3.36 \times 10^{7} \mathrm{~V} / \mathrm{cm}$.) The highest closed channel, $n_{0}$, is determined by the dynamical threshold condition, $n_{0}=$ $\left[\left(|\operatorname{Re} \epsilon|+U_{p}\right) / \omega\right]$ (where $[x]$ is the largest integer $\left.\leq x\right)$, and hence $n_{0}$ is sensitive to both the frequency and the intensity. For open channels $\left(n>n_{0}\right), \operatorname{Re} k_{n}>0$, whereas for a closed channel $m\left(m \leq n_{0}\right)$, the branch of the square root for $k_{m}$ is defined by the condition $\operatorname{Im} k_{m}<0$.

Formal scattering theory for multichannel problems shows that at a particular CC, the partial cross sections for all open channels exhibit TAs $[9,12,13]$. These TAs are caused by interchannel couplings and have a purely quantum origin; thus, they do not have intuitive or quasiclassical interpretations. Moreover, TAs may induce either an increase or decrease of open channel cross sections at a particular CC. The kinds and magnitudes of TAs depend on the potential involved. For a short-range potential, including a ZRP, cusp or step features appear in open channel partial cross sections at a CC [9]. For a Coulomb potential, steplike jumps appear $[12,13]$. To illustrate for our case of a ZRP, consider the amplitude $\mathcal{A}_{n}(F, \omega)$ in Eq. (2). $\mathcal{A}_{n}(F, \omega)$ is nonanalytic at each combination of $F$ and $\omega$ such that $E_{m} \equiv k_{m}^{2}=\epsilon+m \omega-U_{p}=0$, for $m=1,2, \ldots, n$. For the ZRP, these nonanalyticities are branch points of the kind $\left(E_{m}\right)^{l+1 / 2}$, where $l$ is the electron's angular momentum after absorption of $m$ photons. According to dipole selection rules for the initial $s$ electron, $l$ takes values allowed by $0 \leq l \leq m$ and $(-1)^{l+m}=1$ for the case of linear or elliptical polarization; for circular polarization, $l=m$. As a result of interference between singular and regular parts of $\mathcal{A}_{n}$, the rate $\Gamma^{(n)}$ involves singular terms, $c_{n}\left(\left|E_{m}\right|\right)^{l+1 / 2}$, with different coefficients $c_{n}$ below $\left(\operatorname{Im} k_{m}<0\right)$ and above $\left(\operatorname{Re} k_{m}>0\right)$ the $m$ th threshold. Obviously, these TAs are greatest for linear polarization in the vicinity of an even$m$ threshold, when the contribution of the $s$-wave branch point, $\sqrt{E_{m}}$, in $\mathcal{A}_{n}(F, \omega)$ is dominant and the singular term in $\Gamma^{(n)}$ has the form

$$
\Delta \Gamma_{\text {sing }}^{(n)} \propto c_{n} \sqrt{\left|E_{m}\right|} .
$$

Since, in addition to $\Delta \Gamma_{\text {sing }}^{(n)}$, the rate $\Gamma^{(n)}$ involves a positive "regular" part, one can find in this case four "cusp" or "step" threshold signatures superimposed on the regular part of the rate, corresponding to the four possible combinations of the signs of $c_{n}$ above and below threshold [9]. These general statements may be verified from our exact ZRP results for $\Gamma^{(n)}$ in Eqs. (1) and (2). The Bessel functions $J_{2 s+n}$ in (2) are nonanalytic only at $\left|E_{n}\right|=\left|k_{n}^{2}\right| \rightarrow 0$. The nonanalyticities at $E_{m}=0$ for $m=1, \ldots,(n-1)$ are hidden in the coefficients $\phi_{s}$. The existence of these nonanalyticities is obvious from the explicit expressions for matrix elements which enter the equations satisfied by $\phi_{s}$ [15]: these matrix elements may be expressed as absolutely convergent series expansions in terms having the branch point factors $\left(E_{p}\right)^{p^{\prime}+1 / 2}$ for various integers $p$ and $p^{\prime}$.

TAs in the ATD rates $\Gamma^{(n)}$ vanish in the Keldysh [19] approximation: coefficients $\phi_{s}$ for this case reduce to Bessel functions $J_{s}\left[-U_{p} /(2 \omega)\right]$, which are analytic in $F$ and $\omega$. This is not surprising, since neglect of the atomic potential in the intermediate and final states of the escaping electron is equivalent to disregarding channel couplings in a multichannel problem, in which case the TAs obviously disappear. For a ZRP, the channel couplings due to the binding potential interaction are realized only through (intermediate) $s$ states of an escaping electron, since the scattering phases $\delta_{l}(E)$ in this model are zero for $l>0$. In particular, this explains the absence of any TAs for the case of circular polarization (because dipole selection rules forbid any intermediate or final $s$ states). Note also that, for a short-range potential, TAs are clearly distinct at the closure of even- $m$ and odd- $m$ ATD channels. Closing of an even- $m$ channel induces singularities of the first derivative of $\Gamma^{(n)}$ in the energy $E_{m}$ [cf. Eq. (3)], which can usually be seen in the rates as cusp-type peaks or valleys or step-type increases or decreases about the CC. However, odd- $m$ CC's can induce only singularities of the second derivative (due to the $p$-wave component of the electron's wave function), which are less visible but still present (see, e.g., our Figs. 1 and 2 below). This explains the seemingly mysterious fact that ZRP calculations in [7] can identify clear enhancements only at the CC's of even channels, $m=12$ and 14, and not at $m=13$. Owing to their origin in channel couplings, TA signatures must appear in accurate numerical simulations as well. Thus, we believe the enhancements observed numerically in Ref. [5] probably arise from TA effects. Note that this interpretation is not in conflict with that presented in [5] in terms of the behavior of the time-dependent wave function. Finally, the necessity of a quantum interpretation of ATD plateau enhancements follows also from quasiclassical considerations [6,7]: many electron trajectories contribute near the CC's, which means in fact that the quasiclassical approach is inadequate for this case. 


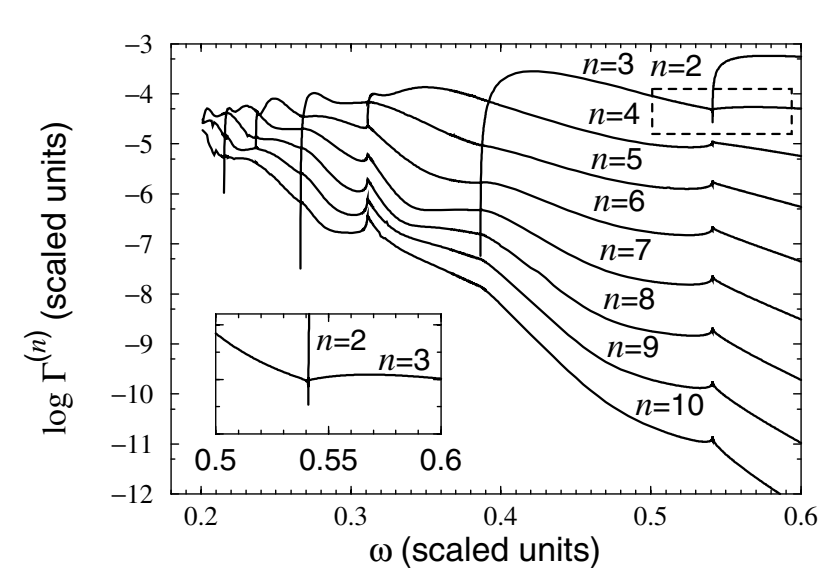

FIG. 1. $n$-photon detachment rates $\Gamma^{(n)}(\omega)$ for laser amplitude $F=0.216$. Inset: Details of $\Gamma^{(3)}$ (on a linear vertical scale) at the closing of the two-photon channel.

In general, the numerical value of the coefficient $c_{n}$ of the $\sqrt{\left|E_{n}\right|}$ term [cf. Eq. (3)] depends upon a complicated interplay of interference between the real and imaginary parts of various terms in Eq. (2) for $\mathcal{A}_{n}$. The perturbation theory (PT) limit of the QQES approach allows an analytic demonstration of this interference. For example, the PT result for $\Gamma^{(3)}$ near the two-photon threshold is [20]:

$$
\Gamma^{(3)}=F^{6}\left[c_{3}^{(0)}+c_{3}^{(1 / 2)} \sqrt{\left|E_{2}\right|}+\mathcal{O}\left(E_{2}\right)\right],
$$

where $E_{2}=2 \omega-\left|E_{0}\right|=2 \omega-1$, and the factors $c_{3}^{(i)}$ are

$$
\begin{aligned}
c_{3}^{(0)} & =\frac{2^{17 / 2}}{945}(197-98 \sqrt{2}), \\
c_{3}^{(1 / 2)} & =\frac{2^{19 / 2}}{135} \begin{cases}28-19 \sqrt{2}, & E_{2}<0 \\
0, & E_{2}>0 .\end{cases}
\end{aligned}
$$

Since the PT amplitude $\mathcal{A}_{3}$ is real for $E_{2} \leq 0$ [18], $c_{3}^{(1 / 2)}$ for $E_{2}>0$ is zero [as it depends on $\operatorname{Im} \mathcal{A}_{3}\left(E_{2}=0\right)$ ]; thus $\Gamma^{(3)}$ is linear in $E_{2}$ above the two-photon threshold. However, the next order PT term for $c_{3}^{(1 / 2)}$ is nonzero and this ensures the expected square root dependence of $\Gamma^{(3)}$ on $E_{2}$ for some small range of positive values [9]. Similar peculiarities appear for other rates $\Gamma^{(n)}$ and their magnitudes and types change as higher order PT terms become important or even dominant (i.e., as PT breaks down). Our exact results for $\Gamma^{(n)}(\omega)(3 \leq n \leq 10)$ in Fig. 1 demonstrate the TAs at the closure of the $n=2$ and $n=4$ channels. We note that clearly visible features around the twophoton threshold appear also in numerical calculations of $\Gamma^{(3)}$ for $\mathrm{H}^{-}$(see, e.g., [21,22]).

In Fig. 2 we plot the rates $\Gamma^{(n)}(F)(8 \leq n \leq 55)$ in the region of the closures of the $n=8,9$, and 10 channels. These data are for intermediate values of the Keldysh parameter, $0.86 \leq \gamma_{K} \leq 1.55$, where $\gamma_{K} \equiv \omega / F$. For $\mathrm{H}^{-}$, our scaled $\omega$ corresponds to the $\mathrm{CO}_{2}$ laser. We observe TAs which are similar to those shown in Fig. 1 for a fixed $F$. However, a striking difference is the large magnitude of the TAs near the $n=8$ and 10 thresholds. Remarkable also is that all four types of TAs predicted by Baz' [9] may be observed. The most remarkable feature of Fig. 2, however, is the concentration of very large magnitude TAs only in a range of $n$ corresponding to the high-energy ATD plateau. We interpret this concentration as resulting from a sensitive interplay of potential- and laser-induced effects at $\gamma_{K} \sim 1$ that occurs only on the plateau. For low $n$, one is in the Keldysh regime in which the laser is treated exactly but the potential only serves to bind the initial state. In the limit of high $n$ (well beyond the plateau), the potential becomes important, but a PT treatment of the laser becomes valid. On the ATD plateau a detailed treatment of the potential is necessary [6] as well as nonperturbative treatment of the laser. In order to obtain TAs in the open channel rates one requires, first, that the laser intensity is sufficiently high to induce (through $U_{p}$ ) the CCs, and, second, that the potential effects in intermediate and final states are included in order to describe the couplings between channels. The plateau region is where the two effects are comparable and the largest TAs are observed.

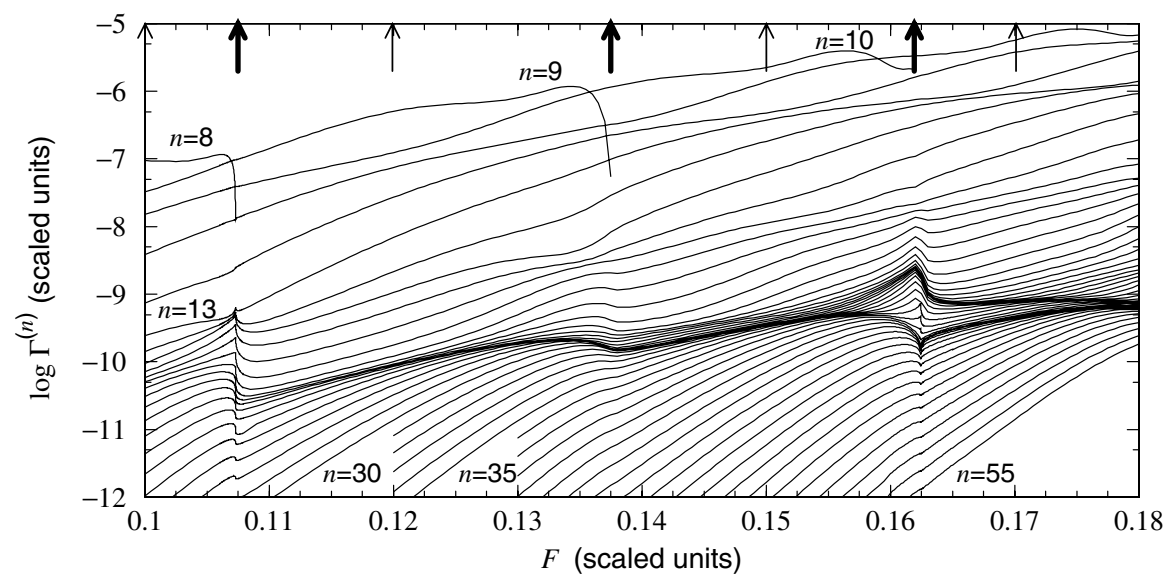

FIG. 2. $\Gamma^{(n)}(F)$ for $\omega=0.155$ in the region where the ponderomotive shift closes the $n=8,9$, and 10 channels. Arrows: values of $F$ at which the corresponding ATD spectra are presented in Fig. 3. The thick arrows indicate the 8, 9, and 10 photon thresholds. 


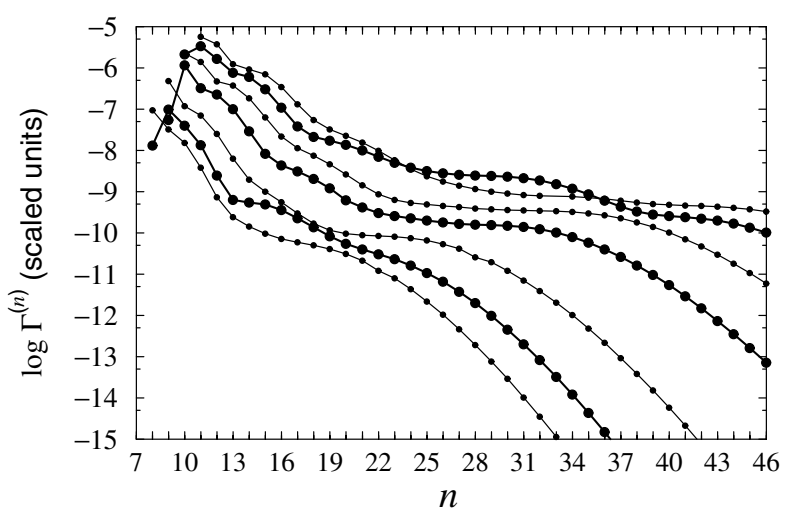

FIG. 3. ATD spectra $\Gamma^{(n)}(8 \leq n \leq 46)$ for $\omega=0.155$ for seven values of the laser amplitude (from bottom to top): $F=$ $0.1,0.1073,0.12,0.1375,0.15,0.162$, and 0.17 . These $F$ values are indicated by arrows in Fig. 2. The dots for $\Gamma^{(n)}$ at fixed $F$ are connected to guide the eye. Bold lines: indicate the $F$ values that close the thresholds for $n=8$ (lower), $n=9$ (middle), and $n=10$ (upper). Note the peak enhancements that appear at the closing of the $n=8$ and 10 channels (i.e., $13 \leq n \leq 19$ for $F=0.1073$ and $26 \leq n \leq 35$ for $F=0.162$ ).

How the TAs shown in Figs. 1 and 2 (as functions of $\omega$ and $F$ ) affect the ATD spectrum is demonstrated in Fig. 3 for 7 values of $F$ indicated by arrows in Fig. 2. The second curve from the bottom, for the $F$ value corresponding to the closure of the $n=8$ channel, demonstrates clearly a resonant-like enhancement for $13 \leq n \leq 19$ compared with the ATD spectra for higher or lower values of $F$. Similarly, the second curve from the top, corresponding to the closure of the $n=10$ channel, demonstrates a similar enhancement for $26 \leq n \leq 35$. Similar enhancements can be observed if one fixes $F$ and varies $\omega$ across the same CCs providing the possibility for experimental observations of the same plateau structures by changing either the intensity or the frequency (or both).

Just as our essentially exact predictions for enhancements of ATD spectra have been traced to TAs at CCs that are well known in multichannel problems involving a short-range potential [9], we expect that a similar explanation applies to the observed enhancements in ATI spectra $[3,4,7,8]$. TAs in systems having a long-range Coulomb field are different: whereas the total cross section is constant across a threshold, the partial rates can exhibit steplike increases or decreases [12]. These steplike features are the analogs of the TAs appearing for a short-range potential [9]. The great similarity of the enhancements in our ATD spectra in Fig. 3 to those observed in the ATI spectra of Ar in Fig. 1 of Ref. [7] imply that these enhancements have a common origin in analytically known TAs near CCs. For Ar (having $\left|E_{0}\right|=15.76 \mathrm{eV}$ ), our scaled $\omega=0.155$ corresponds to $\lambda \approx 500 \mathrm{~nm}$ and our unit of $F$ is $F_{0}=3.2 \times 10^{9} \mathrm{~V} / \mathrm{cm}$.

In conclusion, we have shown both analytically and numerically that the threshold phenomena typical for multichannel problems have great importance in laser detachment of negative ions as thresholds are crossed by changing either laser frequency or intensity in the nonperturbative regime. These TAs offer a clear (and familiar) explanation of recent simulations that have predicted resonance-like enhancements of high-order ATI and ATD peaks, and especially of experimental observations of such enhancements of ATI spectra for rare gases.

This work was supported by NSF Grant No. PHY0070980, by RFBR Grant No. 00-02-17843, and by Grant No. E00-3.2-515 of the Russian Ministry of Education.

*On leave from Institute for Space Sciences, BucharestMagurele 76900, Romania.

[1] L. F. DiMauro and P. Agostini, Adv. At. Mol. Phys. 35, 79 (1995).

[2] M. Protopapas et al., Rep. Prog. Phys. 60, 389 (1997).

[3] P. Hansch et al., Phys. Rev. A 55, R2535 (1997).

[4] M. P. Hertlein et al., J. Phys. B 30, L197 (1977).

[5] H. G. Muller and F. C. Kooiman, Phys. Rev. Lett. 81, 1207 (1998); H. G. Muller, Phys. Rev. A 60, 1341 (1999).

[6] R. Kopold and W. Becker, J. Phys. B 32, L419 (1999).

[7] G. G. Paulus et al., Phys. Rev. A 64, 021401(R) (2001).

[8] E. Cormier et al., J. Phys. B 34, L9 (2001).

[9] A. I. Baz', Zh. Eksp. Teor. Fiz. 33, 923 (1957) [Sov. Phys. JETP 6, 709 (1958)]; L. D. Landau and E. M. Lifshitz, Quantum Mechanics (Pergamon, Oxford, 1977), Sec. 147.

[10] F. H. M. Faisal and P. Scanzano, Phys. Rev. Lett. 68, 2909 (1992).

[11] W. Becker et al., Phys. Rev. A 46, R5334 (1992).

[12] L. Fonda and R. G. Newton, Ann. Phys. (Paris) 7, 133 (1959); L. Fonda, Suppl. Nuovo Cimento 20, 116 (1961).

[13] M. Gailitis, Zh. Eksp. Teor. Fiz. 44, 1974 (1963) [Sov. Phys. JETP 17, 1328 (1963)].

[14] N. L. Manakov et al., Phys. Rep. 141, 319 (1986).

[15] N. L. Manakov and A. G. Fainshtein, Zh. Eksp. Teor. Fiz. 79, 751 (1980) [Sov. Phys. JETP 52, 382 (1980)].

[16] N. L. Manakov et al., J. Phys. B 33, R141 (2000).

[17] M. V. Frolov et al., J. Phys. B 34, L579 (2001).

[18] B. Borca et al., Phys. Rev. Lett. 87, 133001 (2001).

[19] L. V. Keldysh, Zh. Eksp. Teor. Fiz. 47, 1945 (1964) [Sov. Phys. JETP 20, 1307 (1965)].

[20] For the full PT result for $\Gamma^{(3)}$, see Eq. (20) of Ref. [15].

[21] D. Proulx et al., Phys. Rev. A 49, 1208 (1994).

[22] L. A. A. Nikolopoulos and P. Lambropoulos, Phys. Rev. A 56, 3106 (1997). 